\title{
HIGH HEAT FLUX EVAPORATIVE NANOPOROUS SILICON MEMBRANE DEVICE FOR ADVANCED THERMAL MANAGEMENT
}

\author{
Daniel F. Hanks ${ }^{l}$, Jay Sircar ${ }^{l}$, Zhengmao Lu ${ }^{l}$, Dion S. Antao ${ }^{l}$, Kevin R. Bagnall, \\ Banafsheh Barabadi ${ }^{1}$, Todd R. Salamon ${ }^{2}$, and Evelyn N. Wang*l \\ ${ }^{1}$ Massachusetts Institute of Technology, Cambridge, Massachusetts, USA \\ ${ }^{2}$ Bell Labs, Alcatel-Lucent, New Providence, New Jersey, USA
}

\begin{abstract}
We report the design, fabrication and characterization of a novel nanoporous membrane evaporation device to address ultrahigh heat fluxes in advanced electronics. Our design leverages high capillary pressures $(\sim 500 \mathrm{kPa})$ generated by a liquid wetting $130 \mathrm{~nm}$ diameter pores of an ultra-thin $(600 \mathrm{~nm})$ silicon membrane, suspended over a microfluidic supply network. The device was microfabricated in silicon and tested using methanol as the working fluid. We demonstrated a heat dissipation over $600 \mathrm{~W} / \mathrm{cm}^{2}$ with a heat transfer coefficient more than $15 \mathrm{~W} / \mathrm{cm}^{2} \mathrm{~K}$, which suggests this approach is promising as a high flux thermal management strategy.
\end{abstract}

\section{INTRODUCTION}

Heat dissipation is a critical limitation in a range of electronic devices including microprocessors, solar cells, laser diodes and power amplifiers. Advanced thermal management solutions using phase change heat transfer are one of the most promising approaches to address these challenges. While liquid-vapor phase change techniques such as flow boiling in microchannels have been thoroughly investigated, significant limitations associated with flow instabilities and power consumption prohibit practical implementation [1, 2]. Meanwhile, capillary driven evaporation structures such as those used in vapor chambers and heat pipes, e.g. sintered particles, are limited by capillary dryout. This process occurs when the viscous pressure resistance overcomes the capillary pumping potential which leads to the wick drying out. Water is the highest performing fluid in traditional capillary pumped wicks due to its high surface tension and high latent heat relative to its viscosity. Since the capillary and viscous pressures are coupled to pore size, the highest critical heat flux (CHF) are for wicks with particle sizes of $250-355 \mu \mathrm{m}$ using water and yield $\sim 500 \mathrm{~W} / \mathrm{cm}^{2}$ during nucleate boiling in the wicking structure but less than $100 \mathrm{~W} / \mathrm{cm}^{2}$ in pure evaporation [3], i.e., without nucleate boiling. The heat transfer coefficient in pure evaporation is low as a result of the conduction resistance through the wick which is typically 0.6$1.2 \mathrm{~mm}$ thick. Advancements have been made using bi-porous wicks which leverage high capillarity pores with high permeability mesh [4-7], however the thickness of the structures $(135-250 \mu \mathrm{m})$ still limits the heat transfer coefficient before the onset of boiling.

In this work, we designed a device where the wicking structure has a thickness of only $2.6 \mu \mathrm{m}$ and consists of a suspended nanoporous membrane in which high capillarity pores $(130 \mathrm{~nm}$ diameter, $0.6 \mu \mathrm{m}$ long) are bonded to a high permeability liquid supply network of channels that are $2 \mu \mathrm{m}$ wide, $2 \mu \mathrm{m}$ high and $200 \mu \mathrm{m}$ long (Figure 1). Each section of the membrane is $200 \mu \mathrm{m}$ wide, $10 \mathrm{~mm}$ long and bonded to the liquid supply channels which are connected to a manifold. The liquid entering the device is distributed by the manifold which is $100 \mu \mathrm{m}$ wide and $10 \mathrm{~mm}$ long. With an alternating array of membrane and manifold channels, areas larger than $1 \mathrm{~cm}^{2}$ can be cooled. Some of the liquid bypasses the device while some liquid is drawn into liquid supply microchannels by the capillarity of membrane pores where it evaporates from the nanoporous membrane surface. This configuration leverages the passive operation and stable, self-regulating nature of heat pipes while extending the heat flux in the pure evaporation regime by $10 \times$ compared to traditional wicks. The capillary pressure generated by $130 \mathrm{~nm}$ pores is large enough to reach $\sim 1000 \mathrm{~W} / \mathrm{cm}^{2}$ even with dielectric fluids which exhibit low surface tension and low latent heat compared to water. Despite the lower latent heat compared to water, dielectric fluids are generally highly volatile which results in a higher heat transfer coefficient. Furthermore, the use of dielectric

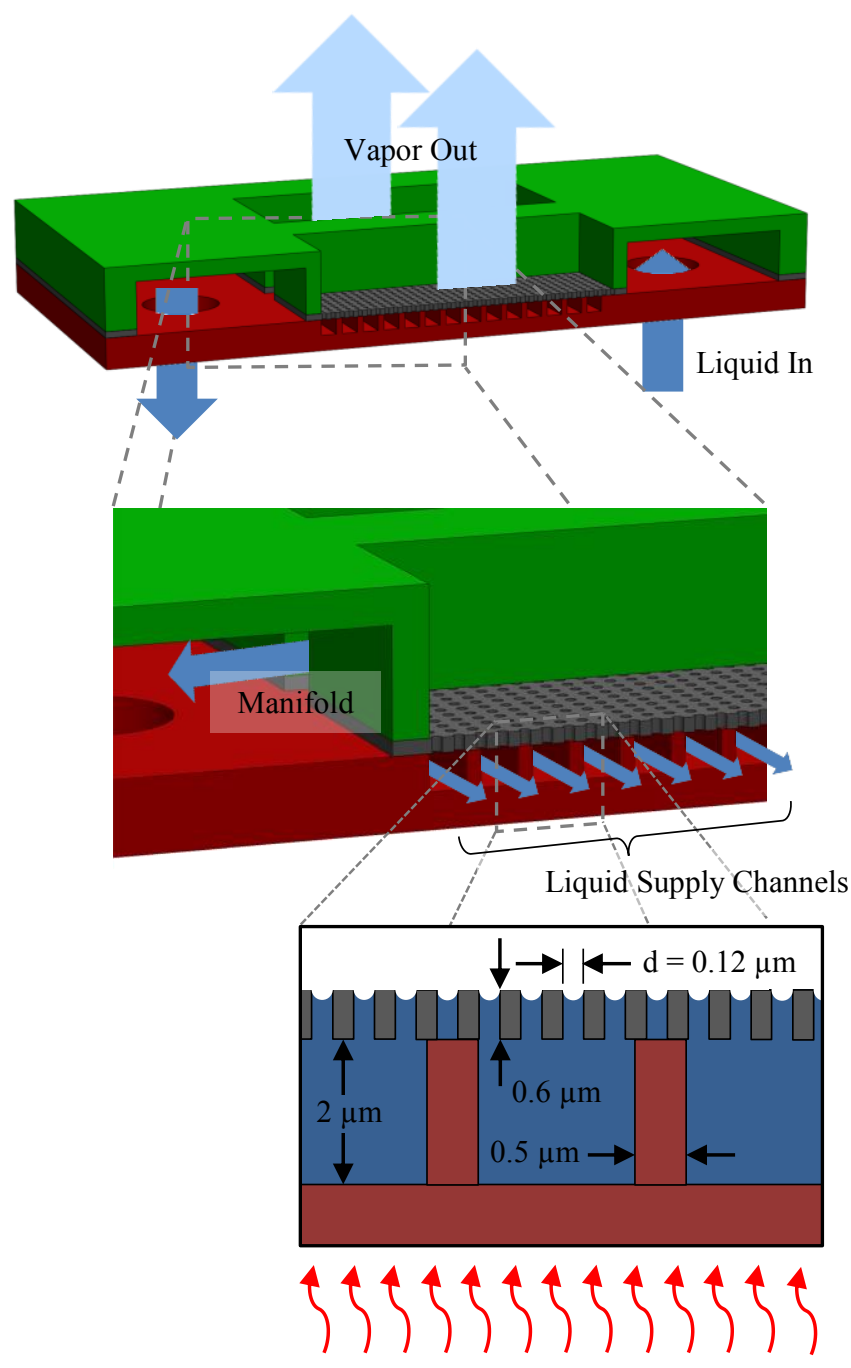

Figure 1: Cross-sectional schematic of supported membrane structure. Liquid flows through a series of manifold channels (shown in green) and is wicked into the liquid supply channels (shown in red) before flowing through the membrane pores and evaporating from the liquid-vapor interface. 
fluids is advantageous for cooling power amplifiers because the circulation of an electrically conducting liquid causes a magnetic field which interferes with electronic performance.

Evaporation from nanopores has been investigated experimentally in previous work. Xiao et. al. demonstrated sustained negative liquid pressures in $150 \mathrm{~nm}$ alumina membrane pores during evaporation with isopropyl alcohol [8]. Narayanan et. al. demonstrated heat dissipation of $600 \mathrm{~W} / \mathrm{cm}^{2}$ for hot spots using 50 $100 \mathrm{~nm}$ alumina pores and FC-72 as the working fluid, but the heat transfer coefficient was limited by conduction through a liquid film under the membrane [9]. In the present study, the membrane is supported by a high conductivity structure which also defines the liquid microchannel supply network. Using this design, the suspended membrane structure can be scaled to areas larger than $1 \mathrm{~cm}^{2}$ and achieve higher heat transfer coefficients compared to thicker wicks while dissipating even higher heat fluxes. While we have previously developed a model to predict the performance of evaporation from our suspended membranes [10-12], here we demonstrate the successful fabrication and experimental validation of our proposed design.

\section{FABRICATION}

We fabricated the device using a two-wafer stack consisting of an SOI wafer bonded to a silicon wafer (Figure 2). Pore size uniformity is critical for evaporation from nanoporous membranes because the largest pore limits the maximum capillary pressure. Meanwhile, the interfacial area for small pores is lower which decreases the heat transfer coefficient. Among lithography techniques for patterning nanopores, electron beam lithography is too slow to cover $1 \mathrm{~cm}^{2}$ and block copolymers have too many defects. We chose interference lithography since it is well suited

(a)

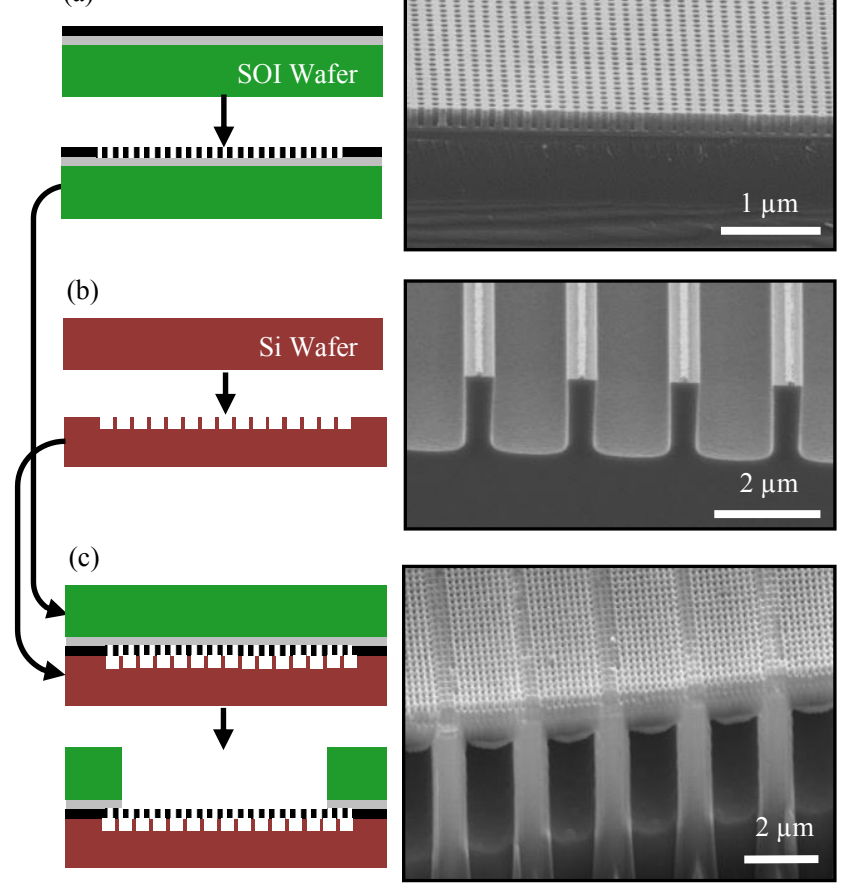

Figure 2: a) Pores defined with interference lithography and etched into SOI wafer. b) Microchannels defined with projection photolithography and etched into the silicon wafer. c) Cross-section of suspended membrane structure after bonding wafers and deep reactive ion etching through the backside of the SOI wafer.

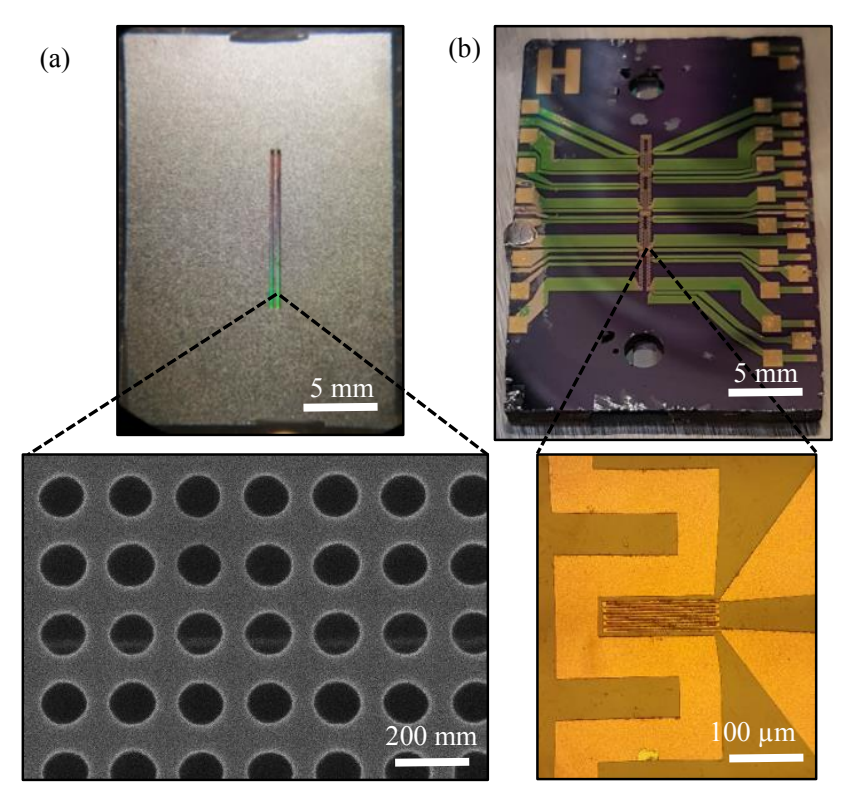

Figure 3: a) Top down view of sample with magnified view of suspended nanoporous membrane. The supporting ridges are faintly visible through the membrane pores. b) Back side of sample with magnified view of RTD within serpentine heater.

based on its high uniformity, low defect density, and fast exposure time. We first defined a pattern for $130 \mathrm{~nm}$ holes on the device layer (black), with a $200 \mathrm{~nm}$ pitch using a $325 \mathrm{~nm} \mathrm{HeCd}$ laser for interference photolithography. The silicon device layer was selectively etched to high aspect ratio $(5: 1)$ with reactive ion etch using $\mathrm{HBr}+\mathrm{O}_{2}$ chemistry (Figure 2a) using the buried oxide as an etch stop. Also on the SOI wafer, the manifold was etched through the device layer, buried oxide and $140 \mu \mathrm{m}$ into the handle layer (green). On the silicon wafer (red), we etched trenches, which serve as liquid supply microchannels. The walls between the trenches are $0.5 \mu \mathrm{m}$ thick and serve as structural support as well as a thermal conduction pathway for the nanoporous membrane (Figure 2b). Smooth sidewalls were essential in the microchannels to prevent nucleation, therefore, the trenches were dry etched using a continuous flow of $\mathrm{SF}_{6}+\mathrm{C}_{4} \mathrm{~F}_{8}$ rather than alternating the chemistry which produces scalloped sidewalls. Next the SOI wafer and silicon wafer were fusion bonded together to form a monolithic evaporation structure. Once bonded, the membrane was released by etching through the backside of the SOI using the buried oxide as an etch stop (Figure 2c). The buried oxide (gray) was then dry etched with $\mathrm{CHF}_{3}$ which has high selectivity to silicon dioxide. Finally, platinum heaters and resistive temperature detectors (RTDs) were deposited $200 \mathrm{~nm}$ thick by electron beam evaporation and liftoff to emulate the high performance IC and measure the device temperature during experiments, respectively. Gold was deposited over electrical contact pads and metal traces to improve localization of heating. Silicon nitride was deposited using PECVD below the platinum and above the gold for electrical insulation. The liquid inlet and outlet ports were etched through the silicon wafer to interface with the liquid manifold.

The sample used for experiments has two membrane sections, which are spaced $200 \mu \mathrm{m}$ apart, $10 \mathrm{~mm}$ long and each nominally $200 \mu \mathrm{m}$ wide (Figure 2a) although the actual width is only $162 \mu \mathrm{m}$ due to etch non-uniformity through the handle layer (Figure 2c). The platinum heaters, shown in Figure $3 \mathrm{~b}$, are $200 \mu \mathrm{m}$ wide and located directly underneath each patch of membrane such that the 


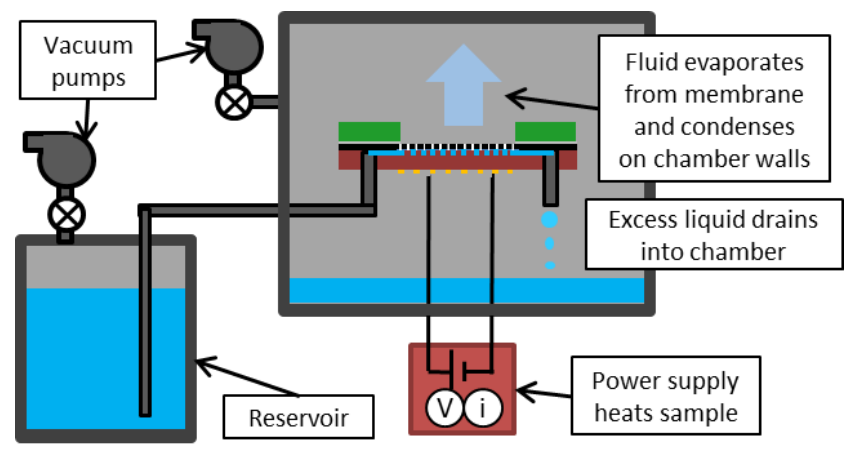

Figure 4: Experimental setup schematic. Degassed liquid is delivered to the device from a reservoir. Liquid is siphoned into the microchannels by capillarity of the pores, evaporates at the membrane surface and condenses on the chamber walls.

total heated area is $0.04 \mathrm{~cm}^{2}$. The average pore diameter is $135.7 \mathrm{~nm}$ and the membrane porosity is 0.378 (Figure $3 \mathrm{a}$ ).

\section{EXPERIMENTAL SETUP}

We experimentally characterized the devices in an environmental chamber that can be pumped to vacuum $(\sim 3$ mTorr $)$ and then back-filled with pure vapor as shown in Figure 4. Methanol was chosen as a working fluid for this work because its surface tension $(0.021 \mathrm{~N} / \mathrm{m})$, viscosity $\left(443 \times 10^{-6} \mathrm{~Pa} \cdot \mathrm{s}\right)$ and latent heat capacity $(1145 \mathrm{~kJ} / \mathrm{kg})$ at $40^{\circ} \mathrm{C}$ are similar to dielectric fluids such as pentane and R245fa. Although methanol is not a dielectric liquid, it is less flammable than pentane and has a lower vapor pressure than R245fa such that it is stable as a liquid at room temperature. The liquid was degassed by pulling vacuum on the reservoir then the liquid was distilled by vaporizing it in one reservoir and the condensing the vapor into a second reservoir. The liquid methanol was then delivered to the sample by heating the reservoir to pressurize it. Heat applied to the sample was measured using current and voltage from an external power supply, although not all of the applied heat was dissipated by evaporation. The time-dependent parasitic heat loss to the sample holder was estimated by heating the sample in a dry ambient. The heat loss due to sensible cooling was calculated using the flow rate and temperature change of the liquid flowing through the sample. The remainder was dissipated by evaporation and is normalized by the heater area to determine the evaporative heat flux. The evaporative heat flux accounts for between $72-91 \%$

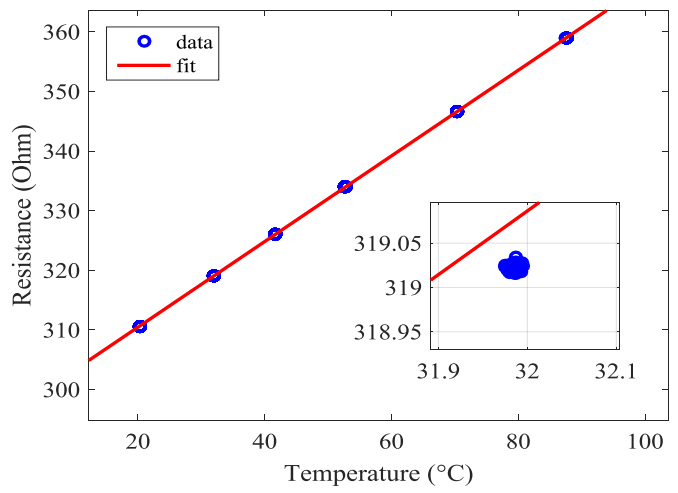

Figure 5: Calibration curve and linear fit for platinum RTDs deposited on the bottom of the sample. The inset shows the data deviate by less than $0.1^{\circ} \mathrm{C}$ from the linear fit.

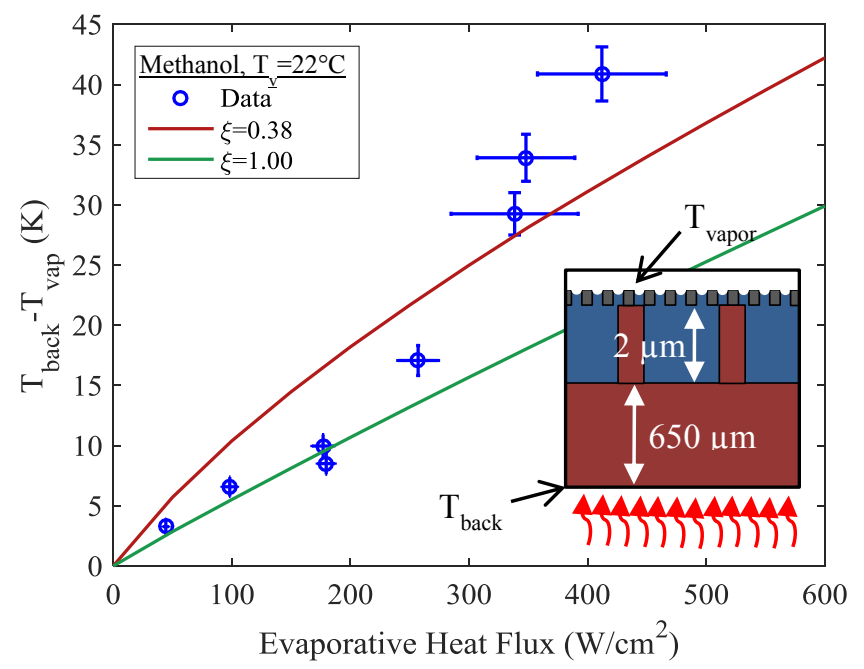

Figure 6: Experimental results of temperature rise as a function of evaporative heat flux. Modeling predictions account for conduction resistance in the $650 \mu \mathrm{m}$ substrate, $2 \mu \mathrm{m}$ supported membrane structure and interfacial transport resistance.

of the applied heat flux depending on the substrate temperature and flow conditions.

Before experiments, the platinum RTDs were calibrated inside the environmental chamber by heating and insulating the outside of the chamber with four independent PID controllers. A T-type thermocouple in contact with the sample was used as the reference temperature and the data was fit with a linear regression as shown in Figure 5.

\section{RESULTS}

The plot of temperature rise as a function of heat flux is shown in Figure 6 where the temperature rise is the difference in RTD temperature on the back side and the ambient vapor temperature. The data are shown alongside our developed model $[11,12]$ which captures conduction resistance in the supported membrane structure along with the interfacial resistance. The intrinsic conductivity in the support $(k=39.2 \mathrm{~W} / \mathrm{mK})$ and membrane $(k=18.2 \mathrm{~W} / \mathrm{mK})$ are reduced due to phonon scattering at small length scales. The model accounts for sub-continuum and non-equilibrium transport in the Knudsen layer above the liquid-vapor interface. The model includes two bounds which represent the fraction of liquid covering the membrane surface, $\xi$. The red curve represents the case where the liquid meniscus is confined to the pores, i.e., the liquid area fraction is equal to the porosity, $\xi=0.378$. The green curve represents liquid spreading to cover a larger area fraction up to a limit of $\xi=1$. At low heat fluxes, the radius of curvature of the meniscus is low relative to the pore radius, so the liquid spreads over the membrane surface. Since the liquid interface is a significant thermal resistance to evaporation, the temperature rise is lower when $\xi \rightarrow 1$. As the heat flux increases, the temperature rise increases as liquid recedes into the pore. Above $300 \mathrm{~W} / \mathrm{cm}^{2}$, the data diverged from the model as the membrane began to clog with soluble, nonvolatile contaminants. The error bars in temperature represent the uncertainty in RTD calibration and uncertainty in the reference thermocouple while the error bars in the evaporative heat flux represent uncertainty in calculating the sensible cooling and parasitic loss.

With the enhanced capillarity of sub-micron pores, we experimentally demonstrated dissipation of heat fluxes of $412 \pm 54 \mathrm{~W} / \mathrm{cm}^{2}$ using methanol when normalizing to the heater area as plotted in Figure 6. Normalizing instead by the evaporation area 
and including sensible liquid cooling, the total heat flux was $612 \pm 28 \mathrm{~W} / \mathrm{cm}^{2}$ with a temperature rise of $40.9 \pm 2.2 \mathrm{~K}$ including the substrate conduction resistance. The highest heat transfer coefficient was $16.0 \pm 2.3 \mathrm{~W} / \mathrm{cm}^{2} \mathrm{~K}$ at $274 \pm 10 \mathrm{~W} / \mathrm{cm}^{2}$ including the substrate conduction resistance. In electronics cooling applications, the substrate may be thinned from $650 \mu \mathrm{m}$ to less than $100 \mu \mathrm{m}$, significantly improving the heat transfer coefficient.

\section{CONCLUSIONS}

We designed, fabricated, and characterized a high performance suspended nanoporous membrane device. This was accomplished with an ultra-thin wicking structure which leverages the high capillarity of $130 \mathrm{~nm}$ pores coupled with a high permeability microfluidic network. Ultimately, the heat flux was limited by accumulation of contaminants in the working fluid, not by capillarity of the nanopores. In the future, the liquid channels can be configured to constantly flush contaminants out of the sample. This work represents a new paradigm for phase-change heat transfer and promises a new thermal management approach for high power density electronics.

\section{ACKNOWLEGMENTS}

The authors acknowledge the funding support from the DARPA ICECool Fundamentals program with Dr. Avram Bar-Cohen as the program manager. Travel support has been generously provided by the Transducer Research Foundation.

\section{REFERENCES}

1. S.G. Kandlikar, "Fundamental issues related to flow boiling in minichannels and microchannels", Experimental Thermal and Fluid Science, 2002. 26(2): p. 389-407.

2. T. Zhang, "Transient Characteristics and Control of Active Thermal Management Systems", Annual Review of Heat Transfer, 2015. 18.

3. J.A. Weibel, S.V. Garimella, and M.T. North, "Characterization of evaporation and boiling from sintered powder wicks fed by capillary action", International Journal of Heat and Mass Transfer, 2010. 53(19): p. 4204-4215.
4. T. Semenic and I. Catton, "Experimental study of biporous wicks for high heat flux applications", International Journal of Heat and Mass Transfer, 2009. 52(21): p. 5113-5121.

5. D. Ćoso, V. Srinivasan, M.-C. Lu, J.-Y. Chang, and A. Majumdar, "Enhanced heat transfer in biporous wicks in the thin liquid film evaporation and boiling regimes", Journal of Heat Transfer, 2012. 134(10): p. 101501.

6. Q. Cai and C.-L. Chen, "Design and test of carbon nanotube biwick structure for high-heat-flux phase change heat transfer", Journal of Heat Transfer, 2010. 132(5): p. 052403.

7. S.S. Kim, J.A. Weibel, T.S. Fisher, and S.V. Garimella. "Thermal performance of carbon nanotube enhanced vapor chamber wicks". in 2010 14th International Heat Transfer Conference. 2010: American Society of Mechanical Engineers.

8. R. Xiao, S.C. Maroo, and E.N. Wang, "Negative pressures in nanoporous membranes for thin film evaporation", Applied Physics Letters, 2013. 102(12): p. 123103.

9. S. Narayanan, A.G. Fedorov, and Y.K. Joshi, "Gas-assisted thin-film evaporation from confined spaces for dissipation of high heat fluxes", Nanoscale and Microscale Thermophysical Engineering, 2009. 13(1): p. 30-53.

10. D.F. Hanks, Z. Lu, S. Narayanan, K.R. Bagnall, R. Raj, R. Xiao, R. Enright, and E. Wang. "Nanoporous evaporative device for advanced electronics thermal management". in Thermal and Thermomechanical Phenomena in Electronic Systems (ITherm), 2014 IEEE Intersociety Conference on. 2014: IEEE.

11. Z. Lu, S. Narayanan, and E.N. Wang, "Modeling of Evaporation from Nanopores with Nonequilibrium and Nonlocal Effects", Langmuir, 2015. 31(36): p. 9817-9824.

12. Z. Lu, T.R. Salomon, S. Narayanan, K.R. Bagnall, D.F. Hanks, D. Antao, B. Barabadi, J. Sircar, M.E. Simon, and E.N. Wang, "Design and modeling of membrane-based evaporative cooling devices for thermal management of high heat fluxes", IEEE Transactions on Components, Packaging and Manufacturing Technology, In Review, 2016.

\section{CONTACT}

*Evelyn Wang, enwang@mit.edu 